\title{
Comparative Evaluation of the Echocardiographic Parameters of Dogs with Chronic Mitral Valve Regurgitation Treated with Amlodipine or Pimobendan
}

\author{
Orson Nobumitsu Kamakura, Guilherme Teixeira Goldfeder, Paula Hiromi Itikawa, \\ Rebecca Bastos Pessoa \& Maria Helena Matiko Akao Larsson
}

\begin{abstract}
Background: Chronic mitral valve regurgitation is the most common heart disease in dogs. It is caused by myxomatous mitral valve degeneration, an acquired degenerative disease that can lead to congestive heart failure. Pimobendan is a mixed action drug, with inotropic and vasodilator proprieties, widely used to treat such dogs. Amlodipine is a pure vasodilator drug used as an anti-hypertensive agent, with no inotropic effects. This study aimed to compare echocardiographic, electrocardiographic and blood pressure parameters between dogs with congestive heart failure treated with pimobendan and those treated with amlodipine in addition to conventional therapy.

Materials, Methods \& Results: A prospective, randomized, double-blind study was conducted in dogs with stage C chronic mitral valve regurgitation according to the 2009 American College of Veterinary Internal Medicine (ACVIM) guidelines, who weighed up to $15 \mathrm{~kg}$. Randomization was performed using appropriate software and the sealed envelope technique. Blinding of the owner, the main researcher, and the person who performed the clinical analysis was guaranteed by the use of identical capsules and bottles for both pimobendan and amlodipine. Statistical analysis was performed using specialized software. Eligibility was determined by predefined inclusion and exclusion criteria. Dogs with cardiac disease other than mitral and/or tricuspid degeneration, patients already undergoing pharmacological treatment with drugs other than enalapril and furosemide, patients with systolic blood pressure below $100 \mathrm{mmHg}$, patients with chronic kidney disease in stages III or IV according to International Renal Interest Society staging system for chronic kidney disease (2009), and patients with other systemic debilitating diseases were excluded from the study. The animals were randomized into two groups based on the drug they received. Group A received pimobendan $(\mathrm{n}=10)$ and group B received amlodipine $(\mathrm{n}=11)$. All animals were also treated with furosemide and enalapril maleate and evaluated three times over a period of sixty days (T0, T30, T60). Changes in echodopplercardiographic and electrocardiographic parameters and systolic blood pressure were investigated. There were no significant differences in electrocardiographic parameters or systolic blood pressure between the groups at any time. Among the echocardiographic parameters, only early diastolic myocardial peak velocity (Em) and late diastolic myocardial peak velocity (Am) showed a significant difference between groups.

Discussion: This was the first prospective randomized double-blind study comparing pimobendan and amlodipine in the treatment of dogs with stage $\mathrm{C}$ chronic mitral valve regurgitation according to the ACVIM consensus statement. The use of pimobendan in these patients has been debated because myocardial inotropism is generally preserved in them. Some authors argue that early use of the drug can even lead to deleterious effects on the heart.

In the present study, the groups showed no statistically significant differences in systolic blood pressure or electrocardiographic parameters. Among the echocardiographic parameters, statistically significant differences were only observed in the speed of Em waves between groups at T0, in the speed of Em waves in the amlodipine group between T0 and T60 times, and in the speed of Am waves in the amlodipine group between T30 and T60 times. Both parameters are indices of diastolic function and may indicate a change in early myocardial relaxation. From the findings of this study, we could not conclude whether one drug was superior to the other.
\end{abstract}

Keywords: pimobendan, amlodipine, CHF, dogs, mitral. 


\section{INTRODUCTION}

Chronic mitral valve regurgitation (CMVR) is the most frequent heart disease of dogs, accounting for $75-85 \%$ of cases of heart diseases.

Pimobendan is an inodilator drug widely used to treat dogs with chronic heart disease (CHD). It has mixed action, inotropic and vasodilator, acting by calcium sensitization to troponin $\mathrm{C}$ and inhibition of phosphodiesterase, promoting increased cardiac output, reduction both in preload and afterload and increased myocardial contractility without increases in myocardial oxygen and energy consumptions. It's a safe drug with few collateral effects and good therapeutical results in dogs with mitral degeneration and dilated cardiomyopathy $[5,8]$.

Amlodipine is a pure long acting and potent vasodilator, with no inotropic effects. Its widely used in hypertensive patients and also reduces both preload and afterload. It acts by calcium channel blockage. Its use is not a consensus in chronic heart diseases in dogs [1].

Previous study have shown that pimobendan improves quality of life and increases the survival time of dogs with chronic heart disease compared with ACE inhibitors and digoxin $[9,10]$, although no studies have compared the pimobendan response versus amlodipine. The present study aims to compare cardiac parameters of dogs in stage C of ACVIM classification, treated with conventional therapy (furosemide, enalapril) and pimobendan or amlodipine. We hypothetise that, in this stage of disease, a pure vasodilator effect of amlodipine is comparable to the inodilator effect of pimobendan.

\section{MATERIALS AND METHODS}

The study was performed at Veterinary Teaching Hospital of the School of Veterinary and Animal Science of University of São Paulo, between August 2014 and January 2016. All owners received details of the study and participation was voluntary and all owners signed a consent and commitment form.

The study was designed prospectively, doubleblinded, randomized. Only patients on stage $\mathrm{C}$ chronic valvular disease with mitral and / or tricuspid involvement were selected, regardless of sex, breed and age. Patients should weight no more than $15 \mathrm{~kg}$. Dogs with other cardiac diseases, kidney disease in stages III and IV according to IRIS Staging System of CKD, other debilitating systemic diseases, systolic blood pressure below $100 \mathrm{mmHg}$, and patients already receiving pimo- bendam, amlodipine or other drugs besides enalapril and furosemide were discarded.

We evaluated the electrocardiographic parameters, systemic blood pressure and echocardiographic parameters of all patients enrolled, at three different times, T0 or baseline (at the time that the patient adhered to the project), T30 (30 days after initiation of treatment) and T60 (60 days after treatment initiation). The patients were randomized to two groups, Group A (receiving pimobendan) and Group B (receiving amlodipine). Ten and eleven patients composed the groups, respectively. All of the dogs were treated with furosemide in association with enalapril maleate in conventional doses, and pimobendan (VetMedin $\left.{ }^{\circledR}\right)^{1}$ remanipulated at a dose of $0.25 \mathrm{mg} / \mathrm{kg}$ every $12 \mathrm{~h}$, or amlodipine at a dose of $0.1 \mathrm{mg} / \mathrm{kg}$ every $12 \mathrm{~h}$, orally.

Systolic Blood Pressure (SBP) was measured by the non-invasive method of Doppler ultrasonography. The animals were placed in lateral recumbency and the appropriate cuff was placed in their forearm. Cuff size was stablished to be $40 \%$ of the selected forearm circumference. The equipment used was the Parksmedical® Ultrasonography Doppler, model 811-B ${ }^{2}$.

In order to perform the electrocardiography, ECAFIX ${ }^{\circledR}$ (electrocardiographer model ECG 6$)^{3}$ was used. The animals were placed in right lateral recumbence and the bipolar leads I, II and III were recorded, as well as the unipolar leads aVR, aVL and aVF, and the pre-cordials CV5RL (rV2), CV6LL (V2), CV6LU (V4) and V10. The paper speed used was $25 \mathrm{~mm} / \mathrm{s}$ and the calibration was $1 \mathrm{mV} / 10 \mathrm{~mm}$. Lead DII was also recorded in the speed of $50 \mathrm{~mm} / \mathrm{s}$. Electrocardiographic parameters were evaluated according to Tilley [16].

Echocardiographic examinations were performed according to the Echocardiography Committee of the Specialty of Cardiology - American College of Veterinary Internal Medicine [15] and the American Society of Echocardiography (ASE) recommendations $[3,11]$. The equipment used was the portable device Vivid $\mathrm{I}^{\circledR}{ }^{4}$. The animals were not sedated or tranquilized in order to undergo the echocardiographic exam. Simultaneous electrocardiography was performed.

Randomization was performed using appropriate software and the sealed envelope technique. Blinding of the owner, the main researcher and the person who made the clinical analysis was guaranteed by the use of identical capsules and bottles for both 
pimobendan and amlodipine. Statistical analysis was performed using the SPSS $19.0 \AA$ software ${ }^{5}$. ANOVA for repeated measures was used and the data was organized in tables, line graphs and Box Plot charts. To compare means, we used the Student's t test, and $\mathrm{p}$ value $<0.05$ was considered statistically significant.

\section{RESULTS}

Twenty-five patients were screened for the present study. We obtained data from 21 patients, since those dogs whose owners administered medications wrongly or did not attend the return were excluded, as well as animals with pulmonary edema or those who died in the interval between enrolment and beginning of medications.

There was a predominance of male dogs and Poodles in both groups. There was no statistical difference between the weight and age of the animals randomized to each group, as displayed in Table 1. In the analysis of SBP, there was no intragroup difference between the measurements at different times, but in the intergroup analysis, measurements of the PIMO group were higher in all evaluated times. Heart rate, however, has not changed over time or between groups or within the same group.

Regarding the echocardiographic parameters, no difference was observed between groups or within the same group at different times in IVSd, LVPWd, LVIDd, FS, EF, AO and AO/LA.

Regarding the assessment of diastolic function, no differences were observed between peak-A wave nor peak-E wave between groups and within the same group. No differences were detected between the E wave deceleration time either, or IVRT. The peak-Em wave, however, was different between T0 and T60 in the group receiving amlodipine, being lower in the latter time. As regards the Am wave, both groups had lower values in T60 compared to T30.

In the analysis of the speed of transvalvular flows, no difference was observed in measurements of the aortic valve peak velocity or the pulmonary valve peak-velocity, similarly to the speed of mitral regurgitation. There were no differences in the measurement of their gradients too, either in the analysis between groups or in response to time within the same group.

Electrocardiographically, no difference was observed among the groups in any of the segments or evaluated waves.

\section{DISCUSSION}

There are no studies in the literature comparing the effects of amlodipine and pimobendan in dogs with CMVR. Given the high cost of pimobendan in Brazil, it would be interesting to investigate the possibility of using a less expensive drug, which could provide similar clinical benefits. In addition, there is a discussion about the benefit of using an inodilator in symptomatic patients whose ventricular dysfunction is debatable. Thus, this study sought to investigate whether pimobendan and amlodipine would cause similar echocardiographic changes in the animals evaluated.

The study was performed in a double-blind randomized fashion, minimizing the possibility of evaluators tendencies. In addition, the echocardiograms were performed by the same examiner, minimizing variations that could influence the results, as described in the literature [6].

With respect to the epidemiology of CMVR, it is known that males, small animals, and older ones are more frequently affected [7], confirming what was observed in this study. Treatment goals concerning CHF patients are to minimize the consequences of volume overload (reduction of pre and afterload), reduced cardiac work and delay secondary cardiac remodeling [1].

In this study, all of the selected patients were in stage $\mathrm{C}$ according to ACVIM classification, which means they either were or have been decompensated. The progression of the $\mathrm{CHF}$ (staging) during treatment was not the objective of the study. It is known that dogs which progress to $\mathrm{CHF}$ have prognostic dependent factors such commitment of the owner to the treatment, the occurrence of cardiovascular complications such as pulmonary hypertension, cardiac tamponade, arrhythmias or rupture of chordae tendineae and presence of comorbidities, especially chronic kidney disease [4]. In this study, only one patient had a complication by concomitant illness. At 30 and 60 days of treatment, such patients were excluded from the study because they developed important azotemia.

Regarding the profile of the dogs included, not all of them could have the estimated lung pressure gradient assessed. However, of the 21 participating animals, $16(76 \%)$ had estimated pulmonary artery pressure $>40 \mathrm{mmHg}$ on at least one of the evaluations, which represents a significantly higher percentage than the $16 \%$ observed by Serres et al. [12] in 2006. One 
Table 1. Baseline parameters of the experimental groups.

\begin{tabular}{|c|c|c|c|}
\hline Parameter & Group PIMO $(\mathrm{N}=10)$ & Group AMLO $(\mathrm{N}=11)$ & $P$-value \\
\hline Weight (kg) & $7.07 \pm 3.75$ & $7.12 \pm 2.3$ & $P=0.889$ \\
\hline Age (months) & $12.8 \pm 1.22$ & $11 \pm 2$ & $P=0.094$ \\
\hline $\mathrm{SBP}(\mathrm{mmHg})$ & $136.9 \pm 18.13$ & $115.23 \pm 16.47$ & $P=0.041$ \\
\hline Heart Rate (bpm) & $130.70 \pm 16.28$ & $137.18 \pm 24.14$ & $P=0.112$ \\
\hline $\operatorname{IVSd}(\mathrm{cm})$ & $0.56 \pm 0.10$ & $0.54 \pm 0.14$ & $P=0.860$ \\
\hline LVFWd (cm) & $0.56 \pm 0.11$ & $0.56 \pm 0.10$ & $P=0.922$ \\
\hline LVIDd $(\mathrm{cm})$ & $3.86 \pm 0.86$ & $3.77 \pm 0.45$ & $P=0.825$ \\
\hline LVIDds (cm) & $1.91 \pm 0.69$ & $1.75 \pm 0.33$ & $P=0.991$ \\
\hline $\mathrm{EF}$ & $0.82 \pm 0.07$ & $0.84 \pm 0.05$ & $P=0.602$ \\
\hline Delta D (\%) & $50.6 \pm 6.62$ & $52.55 \pm 5.3$ & $P=0.671$ \\
\hline $\mathrm{LA}(\mathrm{cm})$ & $3.28 \pm 0.94$ & $3.15 \pm 0.43$ & $P=0.985$ \\
\hline Ao $(\mathrm{cm})$ & $1.34 \pm 0.25$ & $1.44 \pm 0.14$ & $P=0.536$ \\
\hline LA/Ao & $2.41 \pm 0.23$ & $3.15 \pm 0.43$ & $P=0.771$ \\
\hline Peak-E wave (m/s) & $1.39 \pm 0.30$ & $1.32 \pm 0.20$ & $P=0.949$ \\
\hline Peak-A wave $(\mathrm{m} / \mathrm{s})$ & $0.76 \pm 0.33$ & $0.68 \pm 0.18$ & $P=0.270$ \\
\hline TDE (ms) & $92.99 \pm 19.33$ & $90.36 \pm 15.19$ & $P=0.307$ \\
\hline $\mathrm{E} / \mathrm{A}$ & $2.28 \pm 1.24$ & $2.04 \pm 0.53$ & $P=0.832$ \\
\hline IVRT (ms) & $45.30 \pm 11.79$ & $46.82 \pm 18.99$ & $P=0.459$ \\
\hline E/TRIV & $3.51 \pm 2.26$ & $3.31 \pm 1.51$ & $P=0.376$ \\
\hline Peak-Em wave $(\mathrm{m} / \mathrm{s})$ & $10.76 \pm 3.56$ & $14.73 \pm 3.66$ & $P=0.021 *$ \\
\hline Peak-Am wave $(\mathrm{m} / \mathrm{s})$ & $8.71 \pm 5.11$ & $8.82 \pm 2.82$ & $P=0.877$ \\
\hline $\operatorname{Vmax}$ Ao (m/s) & $1.30 \pm 0.36$ & $1.09 \pm 0.24$ & $P=0.198$ \\
\hline $\operatorname{Vmax} \operatorname{Pul}(\mathrm{m} / \mathrm{s})$ & $0.82 \pm 0.17$ & $0.68 \pm 0.15$ & $P=0.164$ \\
\hline $\operatorname{Vmax} \operatorname{MR}(\mathrm{m} / \mathrm{s})$ & $5.14 \pm 0.71$ & $4.96 \pm 0.30$ & $P=0.131$ \\
\hline
\end{tabular}

*Statistically relevant data.

possible explanation for this difference is the different patient selection criteria used in the studies. No other cardiovascular complications, such as important arrhythmias, tendon chordal rupture, cardiac tamponade were observed.

There were no significant differences within the same group regarding the systolic blood pressure between the time periods, but there were statistically significant differences between groups in the three moments of evaluation, and the amlodipine group had the lowest measurements. Since the groups were different at baseline, when no hypotensive drug was being used, we could not affirm that amlodipine promotes greater drop in systolic blood pressure than pimobendan. It is known that both the use of amlodipine alone and the use of amlodipine associated with enalapril determine significant reductions in SBP [2].

Suzuki et al. $[13,14]$ conducted comparative studies, using respectively two different doses of pimobendan and amlodipine or benazepril. In both studies, the same parameters were evaluated. Like the present study, both amlodipine and pimobendan managed to significantly reduce left atrial pressure while benazepril alone did not.

Thus, based on the lack of difference found between the drugs evaluated in this study, we can hypothesize that in stages B and C of MVCR, where theoretically the ventricular inotropism is still preserved, the difference between the cardiovascular 
effects of both pimobendan and amlodipine is not so evident. Thus, we might speculate that the benefit of using pimobendan in these cases is due to its vasodilating effects, rather than its positive inotropic effect.

However, it is known that the use of pimobendan in early stages of CMVR increases survival of treated animals compared to placebo and benazepril $[5,10]$. Therefore, the hypothesis that pimobendan and amlodipine may have comparable effects in treating such a condition must be confirmed by further studies with a longer follow-up time and with the primary endpoint being mortality.

This study has several limitations, and the main one is its relatively small group of patients. Since the small sample size results in low statistical power, negative results may be simply because of chance.

In addition, the small sample size and short follow-up period did not allow us to draw conclusions about the mortality, survival and decompensation rates, as well as the evaluation of quality of life. Moreover, in the present study all of the dogs were privately owned and therefore, did not go through a period of adaptation to the exams performed, such as in Suzuki et al. [13] research. Some of them were too agitated during examinations, what may have interfered with the measurements of SBP. The inclusion of other exams, such as NT-proBNP dosage and chest radiographs controls may have revealed differences that could not be detected by the evaluations performed.

Finally, in order to develop the study in an double-blind fashion, drugs were manipulated in capsules, the pimobendan being from the reference medicinal product (Vetmedin ${ }^{\circledR}$ ) and amlodipine from commercial manipulation pharmacy (Drogavet@ São Paulo). Bioequivalence tests were not performed in the drugs used, and perhaps there might be some degree of difference between the drugs used and reference drugs.
Thus, the present work represents an hypothesis generating study, suggesting the possibility that amlodipine may be used as an alternative to pimobendan at earlier stages of CMVR, because of its comparable vasodilating effects. Survival rates of these patients must be studied in larger clinical trials with longer follow-up periods.

\section{CONCLUSIONS}

Amlodipine and pimobendan treated dogs presented similar echocardiographic parameters, as well as SBP measurements. Therefore, it is speculated that either one of the drugs may be used in stage $\mathrm{C}$ CMVR animals. It is also ventured that pimobendan clinical benefits in this stage of the disease may be due to its vasodilating properties rather than its inotropic effects. The small sample size and short follow-up time prevented us to draw conclusions about survival and quality of life, but these variables should be studied in large equivalence clinical trials in the future.

\section{MANUFACTURERS}

${ }^{1}$ Boehringer Ingelheim. Rheinland-Pfalz, Germany. ${ }^{2}$ Parks Medical Electronics. Aloha, OR, USA.

${ }^{3}$ Ecafix Indústria e Comércio Ltda. São Paulo, SP, Brazil.

${ }^{4}$ General Electric Healthcare. Chicago, IL, USA.

${ }^{5}$ International Business Machine Corp. North Castle, NY, USA.

Acknowledgements. Boehringer Ingelheim for supplying VetMedin® capsules for this study.

Ethical approval. All procedures, treatments and animal care were in compliance with the guidelines of the Comissão de Ética no Uso de Animais - CEUA of the School of Veterinary Medicine and Animal Science, University of São Paulo.

Declaration of interest. The authors report no conflicts of interest. The authors alone are responsible for the content and writing of paper.

\section{REFERENCES}

1 Atkins C., Bonagura J., Ettinger S., Fox P., Gordon S., Haggstrom J., Hamlin R., Keene B., Luis-Fuentes V. \& Stepien R. 2009. Guidelines for the diagnosis and treatment of canine chronic valvular heart disease. Journal of Veterinary Internal Medicine. 23(6): 1142-1150.

2 Atkins C.E., Rausch W.P., Gardner S.Y., Defrancesco T.C., Keene B.W. \& Levine J.F. 2007. The effect of amlodipine and the combination of amlodipine and enalapril on the renin-angiotensin-aldosterone system in the dog. Journal of Veterinary Pharmacology and Therapeutics. 30(5): 394-400.

3 Boon J.A. 2011. The Two-Dimensional Echocardiographic Exam. In: Veterinary Echocardiography. Hoboken: WileyBlackwell, pp.54-138. 
4 Borgarelli M. \& Buchanan J.W. 2012. Historical review, epidemiology and natural history of degenerative mitral valve disease. Journal of Veterinary Cardiology. 14(1): 93-101.

5 Boswood A., Häggström J., Gordon S.G., Wess G., Stepien R.L., Oyama M.A., Keene B.W., Bonagura J., MacDonald K.A., Patteson M., Smith S., Fox P.R., Sanderson K., Woolley R., Szatmári V., Menaut P., Church W.M., O’Sullivan M.L., Jaudon JP.,, Kresken J.G., Rush J., Barrett K.A., Rosenthal S.L., Saunders A.B., Ljungvall I., Deinert M., Bomassi E., Estrada A.H., Fernandez Del Palacio M.J., Moise N.S., Abbott J.A., Fujii Y., Spier A., Luethy M.W., Santilli R.A., Uechi M., Tidholm A. \& Watson P. 2016. Effect of Pimobendan in Dogs with Preclinical Myxomatous Mitral Valve Disease and Cardiomegaly: The EPIC Study-A Randomized Clinical Trial. Journal of Veterinary Internal Medicine. 30(6): 1765-1779.

6 Dukes-McEwan J., Borgarelli M. \& Tidholm A. 2003. Proposed guidelines for the diagnosis of canine idiopathic dilated cardiomyopathy. Journal of Veterinary Cardiology. 5(2): 7-19.

7 Ettinger S.J. 2017. The Physical Examination. In: Ettinger S.J., Feldman E. \& Cote E. (Eds). Textbook of Veterinary Internal Medicine. 7th edn. St. Louis: Elsevier Saunders, pp.4-24.

8 Fuentes V.L., Corcoran B., French A., Schober K.E., Kleemann R. \& Justus C. 2002. A double-blind, randomized, placebo-controlled study of pimobendan in dogs with dilated cardiomyopathy. Journal of Veterinary Internal Medicine. 16(3): 255-261.

9 Larsson M.H.M.A., Schwartz D.S., Goldfeder G.T., de Oliveira V.M.C., Itikawa P.H., Mazini A.M., Melo P.R.R., Machado F.L.A., Júnior F.F.L., Kanayama K.K., Pellegrino A., Daniel A.G.T. \& Ossada R. 2014. Pimobendan improves clinical signs in short term compared to digoxin or placebo in dogs with heart failure due to chronic degenerative mitral valve disease. Acta Scientiae Veterinariae. 42(1): 1-7.

10 Lombard C.W., Jöns O. \& Bussadori C.M. 2006. Clinical efficacy of pimobendan versus benazepril for the treatment of acquired atrioventricular valvular disease in dogs. Journal of the American Animal Hospital Association. 42(4): 249-261.

11 Nagueh S.F., Smiseth O.A., Appleton C.P., Byrd B.F., Dokainish H., Edvardsen T., Flachskampf F.A., Gillebert T.C., Klein A.L., Lancellotti P., Marino P., Oh J.K., Popescu B.A. \& Waggoner A.D. 2016. Recommendations for the Evaluation of Left Ventricular Diastolic Function by Echocardiography: An Update from the American Society of Echocardiography and the European Association of Cardiovascular Imaging. Journal of the American Society of Echocardiography. 29(4): 277-314.

12 Serres F., Chetboul V. \& Tissier R. 2006. Doppler echocardiography-derived evidence of pulmonary arterial hypertension in dogs with degenerative mitral valve disease: 86 cases (2001-2005). Journal of the American Veterinary Medical Association. 229(11): 1772-1778.

13 Suzuki S., Fukushima R., Ishikawa T., Hamabe L., Aytemiz D., Huai-Che H., Nakao S., Machida N. \& Tanaka R. 2011. The Effect of Pimobendan on Left Atrial Pressure in Dogs with Mitral Valve Regurgitation. Journal of Veterinary Internal Medicine. 25(6): 1328-1333.

14 Suzuki S., Fukushima R., Ishikawa T., Yamamoto Y., Hamabe L., Kim S., Yoshiyuki R., Machida N. \& Tanaka R. 2012. Comparative effects of amlodipine and benazepril on left atrial pressure in dogs with experimentally-induced mitral valve regurgitation. BMC Veterinary Research. 8: 166.

15 Thomas W.P., Gaber C.E., Jacobs G.J., Kaplan P.M., Lombard C.W., Vet M., Moise N.S. \& Moses B.L. 1993. Recommendations for Standards in Transthoracic Two-Dimensional Echocardiography in the Dog and Cat. Journal of Veterinary Internal Medicine. 7(4): 247-252.

16 Tilley L.P. \& Smith Jr., F.W.K. 2016. Electrocardiography. In: Smith Jr. F.W.K., Tilley L.P., Oyama M.A. \& Sleeper M.M. (Eds). Manual of Canine and Feline Cardiology. Saint Louis: Elsevier, pp.49-76.

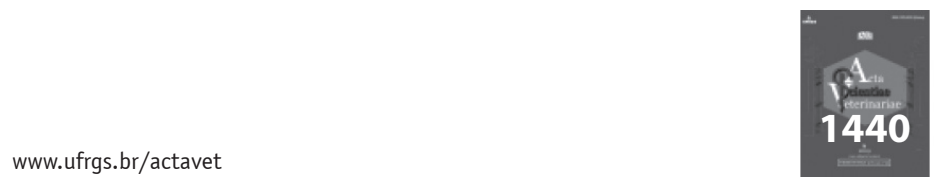

